\title{
Identification of $A N K R D 11$ and ZNF778 as candidate genes for autism and variable cognitive impairment in the novel 16q24.3 microdeletion syndrome
}

\author{
Marjolein H Willemsen ${ }^{\star, 1}$, Bridget A Fernandez ${ }^{2}$, Carlos A Bacino ${ }^{3}$, Erica Gerkes ${ }^{4}$, Arjan PM de Brouwer ${ }^{1}$, \\ Rolph Pfundt ${ }^{1}$, Birgit Sikkema-Raddatz ${ }^{4}$, Stephen W Scherer ${ }^{5}$, Christian R Marshall ${ }^{5}$, Lorraine Potocki ${ }^{3}$, \\ Hans van Bokhoven ${ }^{1}$ and Tjitske Kleefstra ${ }^{1}$
}

The clinical use of array comparative genomic hybridization in the evaluation of patients with multiple congenital anomalies and/or mental retardation has recently led to the discovery of a number of novel microdeletion and microduplication syndromes. We present four male patients with overlapping molecularly defined de novo microdeletions of $16 \mathrm{q} 24.3$. The clinical features observed in these patients include facial dysmorphisms comprising prominent forehead, large ears, smooth philtrum, pointed chin and wide mouth, variable cognitive impairment, autism spectrum disorder, structural anomalies of the brain, seizures and neonatal thrombocytopenia. Although deletions vary in size, the common region of overlap is only $90 \mathrm{~kb}$ and comprises two known genes, Ankyrin Repeat Domain 11 (ANKRD11) (MIM 611192) and Zinc Finger 778 (ZNF778), and is located approximately $10 \mathrm{~kb}$ distally to Cadherin 15 (CDH15) (MIM 114019). This region is not found as a copy number variation in controls. We propose that these patients represent a novel and distinctive microdeletion syndrome, characterized by autism spectrum disorder, variable cognitive impairment, facial dysmorphisms and brain abnormalities. We suggest that haploinsufficiency of $A N K R D 11$ and/or ZNF778 contribute to this phenotype and speculate that further investigation of non-deletion patients who have features suggestive of this $16 \mathrm{q} 24.3$ microdeletion syndrome might uncover other mutations in one or both of these genes.

European Journal of Human Genetics (2010) 18, 429-435; doi:10.1038/ejhg.2009.192; published online 18 November 2009

Keywords: 16q24.3 microdeletion; ANKRD11; ZNF778; cognitive impairment; autism

\section{INTRODUCTION}

Whole-genome scanning technologies such as array comparative genomic hybridization (array CGH) and single-nucleotide polymorphism oligonucleotide arrays (SNP array) have enabled the detection of submicroscopic chromosomal aberrations, which previously escaped detection by routine cytogenetic and molecular cytogenetic techniques. ${ }^{1-3}$ These methods have proven invaluable in the elucidation of genomic regions associated with mental retardation and/or congenital anomalies. ${ }^{4-8}$ Several clinically distinct microdeletion and microduplication syndromes have been reported on the basis of data derived from these techniques, such as the 17q21.31 microdeletion syndrome ${ }^{5}$ and the 1q41-1q42 microdeletion syndrome, ${ }^{9}$ as well as microduplication syndromes involving $7 \mathrm{q} 11.23^{10,11}$ and 17p11.2. ${ }^{12}$ The phenotypic characteristics of microdeletion syndromes can be caused by haploinsufficiency of single genes, for example, TCF4 (MIM 602272) in Pitt-Hopkins syndrome, ${ }^{13}$ EHMT1 (MIM 607001) in the $9 \mathrm{q} 34.3$ subtelomeric deletion syndrome $\mathrm{e}^{14}$ and either CREBBP (MIM 600140) or EP300 (MIM 602700) in Rubinstein-Taybi syndrome. ${ }^{15,16}$ The application of genome-wide array technologies with an increasing density of probes has led to the identification and localization of several genes associated with developmental disorders or abnormal brain development, ${ }^{8}$ including CHD7 (MIM 608892) in CHARGE syndrome ${ }^{17}$ and FOXG1(MIM 164874) in congenital Rett syndrome. ${ }^{18}$ Aberrations of chromosome 16q with clinical relevance have rarely been reported. Before this report, interstitial deletions restricted to band 16q24.3 have not been described. There are only a few reports in medical literature with regard to patients with larger, cytogenetically visible deletions comprising this region, mostly because of an unbalanced complex chromosomal rearrangement. ${ }^{19-29}$ In this study, we aimed to characterize the clinical and molecular features of four patients with submicroscopic interstitial 16q24.3 microdeletions ascertained by genome-wide array analysis and to determine the shortest region of overlap (SRO) to identify candidate genes responsible for their overlapping phenotype.

\section{PATIENTS AND METHODS}

\section{Patient 1}

Patient 1 was ascertained at the age of 22 years when SNP array was performed because of mental retardation, autism spectrum disorder, dysmorphic features and congenital anomalies. He was born after an uncomplicated pregnancy,

\footnotetext{
${ }^{1}$ Department of Human Genetics, Radboud University Nijmegen Medical Centre, Nijmegen, The Netherlands; ${ }^{2}$ Disciplines of Genetics and Medicine, Memorial University of Newfoundland and Provincial Medical Genetic Program, Eastern Health, St John's, Newfoundland and Labrador, Canada; ${ }^{3}$ Department of Molecular and Human Genetics, Baylor College of Medicine, Texas Children's Hospital, Houston, TX, USA; ${ }^{4}$ Department of Genetics, University Medical Centre Groningen, Groningen, The Netherlands; ${ }^{5}$ The Centre for Applied Genomics, The Hospital for Sick Children and University of Toronto, Toronto, Ontario, Canada

*Correspondence: Dr MH Willemsen, 849 Department of Human Genetics, Radboud University Nijmegen Medical Centre, PO Box 9101, Nijmegen 6500 HB, The Netherlands. Tel: +31 243613 946; Fax: +31 243668 753; E-mail: m.willemsen@antrg.umcn.nl

Received 30 June 2009; revised 2 September 2009; accepted 22 September 2009; published online 18 November 2009
} 
induced at 36-week gestation because of maternal preeclampsia. Growth parameters were appropriate for gestational age, with a birth weight between the 50th and 75th percentile. Head circumference was not measured at birth. He was diagnosed with congenital hip dysplasia, but had no other difficulties in the neonatal period. His psychomotor development was delayed. He walked independently at the age of 2.5 years and began using single words after the age of 4 years. At the age of 3 years, he was referred to a pediatric neurologist because of his developmental delay and epilepsy (absence and generalized epilepsy). Cerebral imaging with a computer tomography scan (CT-scan) showed colpocephaly, hypoplasia of the corpus callosum and heterotopia. Vision and audiological evaluations were normal. Routine chromosome analysis showed a normal male karyotype. At the age of 22 years, he was referred for reevaluation. He was moderately mentally retarded and showed behavior consistent with autism spectrum disorder. A Dutch formal test for autism spectrum disorders (AVZ-R 'Autisme- en Verwante stoornissenschaal-ZRevisie', 1999) showed borderline results for the diagnosis of an autism spectrum disorder. His epilepsy was well under control and medical treatment was gradually stopped. On physical examination, he exhibited dysmorphic features, with a high forehead, bitemporal narrowing, long palpebral fissures, large ears, smooth philtrum, wide mouth and micrognathia (Figure 1c and d). He had kyphoscoliosis and strabismus. His height was $175 \mathrm{~cm}$ (3-10th percentile), weight was $64 \mathrm{~kg}$ (50th percentile) and head circumference was $59.5 \mathrm{~cm}$ (90th percentile). Cardiac ultrasound revealed no abnormalities. Bone densitometry showed normal bone density. Genetic diagnostic evaluation with subtelomeric multiplex ligation-dependent probe amplification (MLPA, SALSA MLPA Kit P036, MRC Holland, Amsterdam, The Netherlands) was normal.

\section{Patient 2}

A cursory analysis of patient 2 has been included in a large Canadian autism cohort study, ${ }^{30}$ and here we provide a more detailed clinical and molecular description. Patient 2 was ascertained at the age of 3 years and 3 months when SNP array analysis was performed because of developmental delay, autism spectrum disorder and dysmorphic features. He was born at term after an uncomplicated pregnancy and delivered by cesarean section on maternal indication. He was born with strikingly outstanding and asymmetric ears. On day 3 , he developed low platelets. It was documented as having been caused by alloimmune idiopathic thrombocytopenic purpura (ITP) because of his mother's ITP. After treatment, his platelets remained within the normal range. Psychomotor development was delayed. At 21 months of age, he was unable to walk independently and used three single words with meaning. He was diagnosed with autism by formal tests at the age of 2 years by ADOS- 1 (Autism Diagnostic Observation Schedule) and at the age of 3 years and 4 months by ADI-R (Autism Diagnostic Interview Revised). Psychometric testing at the age of 6 years and 5 months showed average results for nonverbal intelligence; The Naglieri Nonverbal Ability test score was at the 50th percentile and Test of nonverbal intelligence gave a nonverbal IQ of 100. Preschool Language Scale 4 (PLS-4) showed that auditory comprehension was severely delayed (1st percentile) and that expressive communication was moderately delayed (4th percentile). On physical examination, he had a height of $94 \mathrm{~cm}(10-25 \mathrm{th}$ percentile) and weight of $13.5 \mathrm{~kg}$ (10-25th percentile) and a head circumference of $49.5 \mathrm{~cm}$ (50th percentile). Facial dysmorphic features included frontal bossing, long palpebral fissures, a wide mouth with full lips, long and smooth philtrum, anteverted nares, pointed chin and large ears (ears originally outstanding/cupped and asymmetric, now after surgical correction) (Figure 1e). Diagnostic studies, including routine G-banded chromosome analysis, FISH for the Williams syndrome microdeletion and molecular analysis for fragile $\mathrm{X}$ syndrome, were negative. CT-scan of the brain was normal and examination by an ophthalmologist did not show any abnormalities.

\section{Patient 3}

Patient 3 was ascertained at the age of 6 years and 3 months when array CGH analysis was performed because of mental retardation, dysmorphic features and congenital anomalies. He was born at term by vaginal delivery after an uncomplicated pregnancy and his size was appropriate for gestational age. At delivery, he was noted to have a skin rash and hepatosplenomegaly with thrombocytopenia. TORCH (toxoplasmosis, rubella, cytomegalovirus, herpes) infection was excluded and organomegaly and thrombocytopenia were resolved. He suffered a mild intracranial hemorrhage secondary to thrombocytopenia, and was diagnosed with a ventricular septal defect (VSD), patent foramen ovale (PFO), cleft mitral valve and left cryptorchidism in the newborn period. VSD and PFO were repaired at the age of 3 months. Routine G-banded chromosome analysis was normal. At the time of genetics consultation at the age of 6 years and 3 months, he was admitted to hospital for an acute parvovirus myocarditis and dilated cardiomyopathy with severely depressed biventricular function. Further evaluation revealed a history of developmental delay (he walked at the age of 3 years, and spoke his first words at 18-20 months), mental retardation and febrile seizures. On physical examination, he had a height on the 5-12th percentile and a head circumference on the 10th percentile. Dysmorphic features that were observed included a triangular face with a high forehead and frontal bossing, arched eyebrows, large ears, smooth philtrum, wide mouth, pointed chin and high palate (Figure 1f and g). MRI of the brain revealed a posterior fossa arachnoid cyst, thinned corpus callosum, periventricular heterotopias, optic nerve hypoplasia and evidence for an old left hemorrhagic parietal infarct. The heterotopias were unilaterally localized along the lateral margin of the trigone of the right lateral ventricle extending along the temporal horn of the right lateral ventricle. Ophthalmic examination revealed high myopia, intermittent exotropia, horizontal nystagmus, and possible right amblyopia. Audiogram revealed moderate-to-severe bilateral mixed sensorineural and conductive hearing impairment. His complete blood count showed mild macrocytosis (MCV 92.4 FL; normal 76-90), with normal homocysteine and folate values. The cardiomyopathy and macrocytosis resolved, yet he developed complex partial seizures at the age of $71 / 2$ years, with EEG showing a seizure focus of the left temporal region. He continued to make progress in school. He meets criteria for autism under the ADOS.

\section{Patient 4}

Patient 4 was ascertained at the age of 8 years and 10 months when array CGH analysis was performed because of psychomotor retardation, features of autism spectrum disorder and dysmorphic features. He was born at term after an uneventful pregnancy by cesarean section because of breech presentation. His birth weight was $3850 \mathrm{~g}$ (50-90th percentile), but birth length and head circumference are unknown. In the first few weeks, there were feeding problems, probably caused by poor sucking. There was no hypotonia. Psychomotor development was slightly delayed with the child being able to sit independently at 12 months of age and able to walk independently at the age of 18 months. Language development was delayed. Throughout childhood, there were periods of obstipation. There were frequent ear infections. Hearing was tested normal. There is severe bilateral astigmatism. At the age of 7 years and 2 months, verbal IQ was 81 and performal IQ was 67 , as assessed by the WISC III intelligence test. He was diagnosed with an Autism Spectrum Disorder (ASD)/Pervasive Developmental Disorder Not Otherwise Specified (PDD-NOS) by a child psychologist and psychiatrist. There are no signs of epilepsy. On physical examination, he showed dysmorphic features with a high and broad forehead, mildly upslanting palpebral fissures, a double hair whorl, large ears, a preauricular tag and bilateral fusion of the central and lateral incisors (Figure $1 \mathrm{~h}$ and i). His height was $126.3 \mathrm{~cm}$ (10-25th percentile), weight was $23.7 \mathrm{~kg}$ (5-25th percentile) and head circumference was $52.2 \mathrm{~cm}$ (50-75th percentile). Cardiac examination was normal. The left hand showed a single transverse palmar crease. Thumbs were slightly proximally placed. X-rays of the hands showed pseudo-epiphyses of the second metacarpals. Routine G-banded chromosome analysis showed a normal male karyotype. Molecular analysis for fragile X syndrome was negative. Complete blood count showed no abnormalities at the age of 8 years.

\section{Methods}

DNA was obtained from peripheral blood leukocytes and isolated according to standard procedures.

In patient 1 and 2 and both parental couples, a 500K SNP array analysis with the combined two-chip Affymetrix NspI and StyI GeneChip Human Mapping Commercial was performed according to the standard Affymetrix GeneChip protocol (Affymetrix Inc., Santa Clara, CA, USA). For CNV validation in patient 2, multiple SYBR Green-based qPCR assays were used (primer 

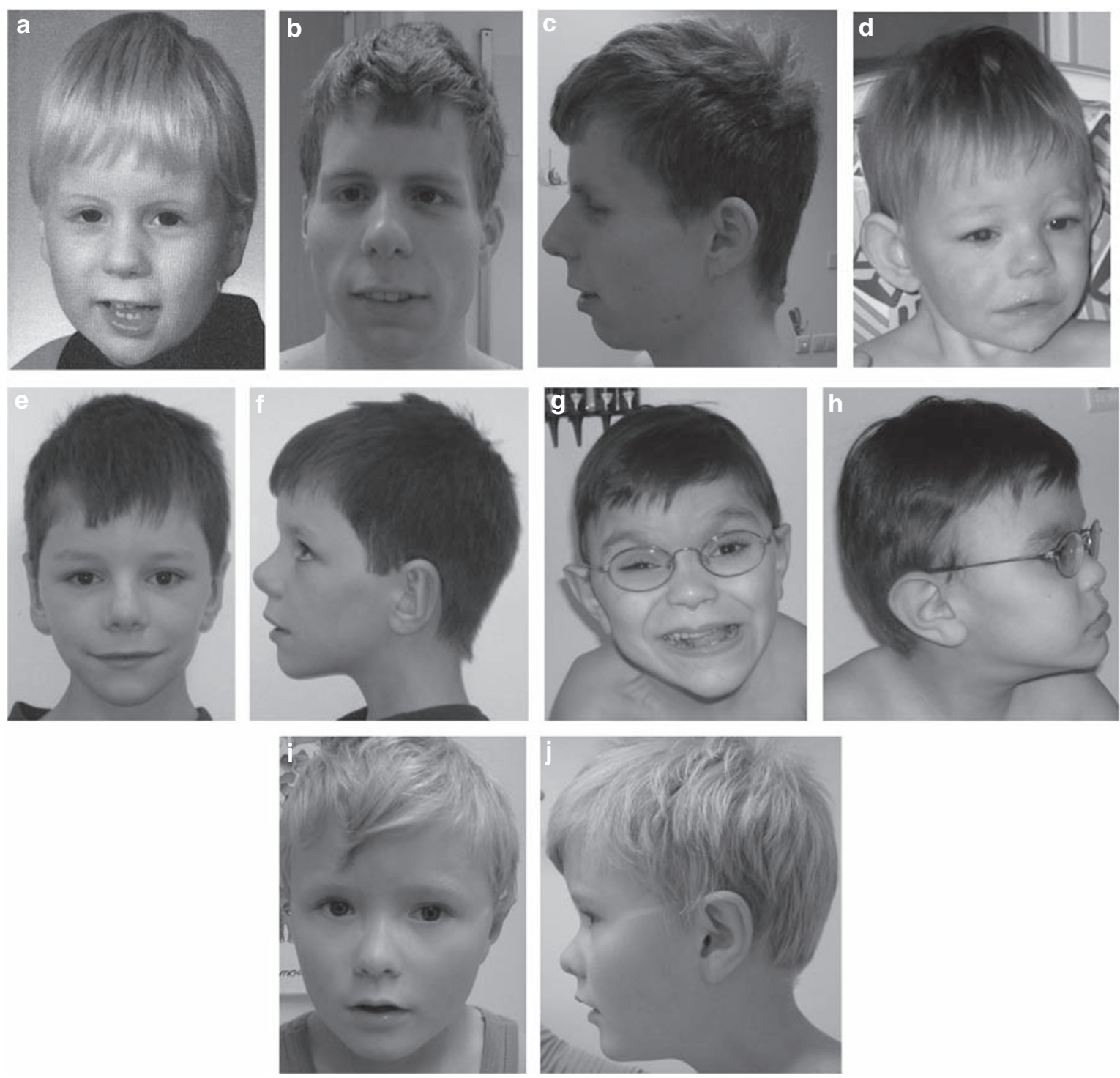

Figure 1 Facial profiles of patients 1-4. (a-c) Patient 1 at several ages, from 6 years (a) to 22 years (b and $\mathbf{c}$ ). (d-f) Patient 2 at the age of 3 years (d) and at the age of 8 years (e and $\mathbf{f}$ ). ( $(\mathbf{g}$ and $\mathbf{h}$ ) Patient 3 at the age of 7 years. (i and $\mathbf{j}$ ) Patient 4 at the age of 8 years and 10 months. Facial features comprise high forehead/frontal bossing and large ears (all patients), broad mouth, long smooth philtrum and pointed chin (patient 1-3). Note the change in facial phenotype in patient 1 at adult age, showing a long and oval face with a full upturned nose, retrognathia and a pronounced groove in the chin.

sequences available upon request) to measure relative copy number in the patient, parents and controls between this chromosome 16q24.3 and a control region (FOXP2). The same approach was used to validate a de novo $500 \mathrm{~kb}$ CNV gain at 3p14.2 near the common fragile site FRA3B in patient 2 . Subsequently, patient 2 was also tested on the Affymetrix 6.0 array.

In patient 3 and his parents, a $244 \mathrm{~K}$ oligo array analysis, according to the Agilent protocol (Agilent Technologies Inc., Santa Clara, CA, USA), was performed.

In patient 4 and his parents, a $105 \mathrm{~K}$ oligo array according to the Agilent protocol was performed (design ID 019015, Agilent Technologies Inc.).

\section{RESULTS}

\section{Clinical features}

A summary of the clinical features of the four patients with a chromosome 16q24.3 microdeletion is shown in Table 1. All patients show a prominent forehead and large ears. Moreover, patients 1-3 share other distinct features (Figure $1 \mathrm{a}-\mathrm{g}$ ), including long smooth philtrum, broad mouth and pointed chin. These were not observed in patient 4 (Figure $1 \mathrm{~h}$ and $\mathrm{i}$ ). In addition to variable cognitive impairment, ranging from moderate mental retardation to normal nonverbal IQ with moderate-to-severe speech delay, all four patients had either autistic features (patient 1) or met the diagnostic criteria for an ASD (patients 2-4). Interestingly, brain abnormalities, including structural anomalies and neuronal migration disorders, were noticed in two of the three patients who underwent brain imaging (patient 1 and 3). Epilepsy and (transient) thrombocytopenia were each noticed in two of the four patients. Nonspecific ocular problems were seen in three of the four patients. One patient, patient 3, with the largest deleted region, had a ventricular septal defect and cleft mitral valve.

\section{Molecular findings}

The de novo $\mathrm{CNV}$ results observed in the four patients are summarized in Table 1 and the overlapping deletions at chromosome 16q24.3 are schematically shown in Figure 2. All deletions were mapped according to the USCS genome browser build May 2004. 
In patient 1 , an interstitial $378 \mathrm{~kb}$ loss was identified (87.65-88.03 Mb): 46, XY.arr snp 16q24.3 (SNP_A-1895824 $\rightarrow$ SNP_A-2058988) $\times 1 \mathrm{dn}$.

In patient 2 , an interstitial loss of $265 \mathrm{~kb}(87.80-88.06 \mathrm{Mb}): 46, \mathrm{XY}$, $16 q 24.3$ (SNP_A-2223520 $\rightarrow$ SNP_A-4274603) $\times 1$ dn was identified. A detailed analysis of the qPCR data in patient 2 revealed that the deletion does not extend to the last exon of $\mathrm{CDH} 15$. According to the assays, coordinates $87788807-87788909$ (Build 36) are not deleted (results not shown). Patient 2 also carried a $500 \mathrm{~kb}$ de novo gain at 3 14.2. An interstitial loss of $2.07 \mathrm{Mb}$ in chromosome band 16q24.2q24.3 was identified in patient $3(86.06-88.13 \mathrm{Mb}$ ) (probe A_16_P40717088 $\rightarrow$ probe A_16_P20559418). In patient 4, an interstitial loss of $1.1 \mathrm{Mb}$ in chromosome band 16q24.2q24.3 was detected $(86.79-87.89 \mathrm{Mb})$ (probe A_14_P119277 $\rightarrow$ A_14_P111955).

In all four cases, analysis of both parents showed normal copy numbers for the deleted regions, suggesting a de novo origin of the deletions. These four chromosome 16q24.3 deletion cases are the only cases that have been detected in a total cohort of more than 3000 patients with developmental delay and/or autism or congenital anomalies. The same region was interrogated over 1000 population controls $^{31}$ and found not to be copy number variable. The Database of Genomics Variants ${ }^{32}$ shows one CNV (Variation_4018) at this site, ${ }^{33}$ involving the first two exons of ANKRD11 but not ZNF778, but it is not found in other studies.

\section{DISCUSSION}

We present detailed clinical and molecular features of four patients with microdeletions within the chromosomal band 16q24.3. The four patients have autistic features or a diagnosis of ASD, variable cognitive impairment and facial dysmorphism, and share a $90 \mathrm{~kb}$ overlapping region comprising two annotated genes, Ankyrin Repeat Domain 11 (ANKRD11) and zinc finger 778 (ZNF778) (Figure 2). The level of cognitive impairment that was observed in these four patients is considerably variable, ranging from moderate mental retardation in patient 1 and 3 to a normal nonverbal IQ with moderate-to-severe

Table 1 Clinical features of the four presented patients with a $16 q 24.3$ microdeletion

\begin{tabular}{|c|c|c|c|c|c|}
\hline Patient & Patient 1 & Patient 2 & Patient 3 & Patient 4 & Total \\
\hline Size of $16 q 24$ deletion & $378 \mathrm{~kb}$ & $265 \mathrm{~kb}$ & $2.07 \mathrm{Mb}$ & $1.1 \mathrm{Mb}$ & 90 kb overlap \\
\hline Mb positions & $87.65-88.03$ & $87.80-88.06$ & $86.06-88.13$ & $86.79-87.89 \mathrm{Mb}$ & SRO: $87.80-87.89 \mathrm{Mb}$ \\
\hline Age at examination & 22 years & 3 years, 3 months & 6 years, 3 months & 8 years, 10 months & Mean age 10 years \\
\hline Cognitive impairment & $\begin{array}{l}\text { Moderate mental } \\
\text { retardation }\end{array}$ & $\begin{array}{l}\text { Normal nonverbal IQ with } \\
\text { moderate-severe speech } \\
\text { delay }\end{array}$ & Moderate mental retardation & $\begin{array}{l}\text { Borderline-normal } \\
\text { verbal IQ mildly } \\
\text { impaired nonverbal IQ }\end{array}$ & $4 / 4$ \\
\hline Autism spectrum disorder & Borderline & + & + & + & $4 / 4$ \\
\hline Height & 3-10th percentile & 10-25th percentile & 5-12th percentile & 10-25th percentile & All (low) normal \\
\hline Head circumference & 90th percentile & 50th percentile & 10th percentile & 50-75th percentile & All normal \\
\hline \multicolumn{6}{|l|}{ Facial characteristics } \\
\hline High forehead & + & - & + & + & $3 / 4$ \\
\hline Frontal bossing & - & + & + & - & $2 / 4$ \\
\hline Bitemporal narrowing & + & - & - & - & $1 / 4$ \\
\hline Long oval face & + & - & - & - & $1 / 4$ \\
\hline Long palpebral fissures & + & + & - & - & $2 / 4$ \\
\hline Arched eyebrows & - & - & + & - & $1 / 4$ \\
\hline Large ears & + & + & + & + & $4 / 4$ \\
\hline Smooth philtrum & + & + & + & - & $3 / 4$ \\
\hline Broad mouth & + & + & + & - & $3 / 4$ \\
\hline Pointed chin & + & + & + & - & $3 / 4$ \\
\hline Micrognathia & + & + & - & - & $2 / 4$ \\
\hline High palate & + & - & + & - & $2 / 4$ \\
\hline Congenital heart defect & - & - & $\begin{array}{l}\text { VSD, PFO and cleft mitral valve } \\
\text { with severe mitral regurgitation }\end{array}$ & NT & $1 / 2$ \\
\hline $\begin{array}{l}\text { Structural brain } \\
\text { malformation }\end{array}$ & $\begin{array}{l}\text { Corpus callosum } \\
\text { hypoplasia, } \\
\text { colpocephaly }\end{array}$ & - & $\begin{array}{l}\text { Corpus callosum hypoplasia, dilated } \\
\text { ventricles, optic nerve hypoplasia }\end{array}$ & NT & $2 / 3$ \\
\hline $\begin{array}{l}\text { Neuronal migration } \\
\text { disorder }\end{array}$ & Heterotopias & $\begin{array}{l}\text { Normal unenhanced CT } \\
\text { cerebrum }\end{array}$ & $\begin{array}{l}\text { Perventricular heterotopias } \\
\text { (unilateral) }\end{array}$ & NT & $2 / 3$ \\
\hline Seizures & $\begin{array}{l}\text { Absences and } \\
\text { generalized }\end{array}$ & - & Partial complex & - & $2 / 4$ \\
\hline Ocular problems & Strabismus & - & $\begin{array}{l}\text { High myopia, astigmatism, } \\
\text { horizontal nystagmus }\end{array}$ & $\begin{array}{l}\text { Severe bilateral } \\
\text { astigmatism }\end{array}$ & $3 / 4$ \\
\hline Hearing loss & - & - & $\begin{array}{l}\text { Mixed sensorineural and } \\
\text { conductive hearing loss }\end{array}$ & - & $1 / 4$ \\
\hline Skeletal anomalies & Kyphoscoliosis & - & - & - & $1 / 4$ \\
\hline Genital anomalies & - & - & Unilateral cryptorchidism & - & $1 / 4$ \\
\hline Hematologic disorder & - & $\begin{array}{l}\text { Neonatal thrombopenia } \\
\text { (resolved) }\end{array}$ & $\begin{array}{l}\text { Thrombopenia, macrocytosis } \\
\text { (resolved) }\end{array}$ & - & $2 / 4$ \\
\hline
\end{tabular}


$16 q 24.2$

$16 q 24.3$

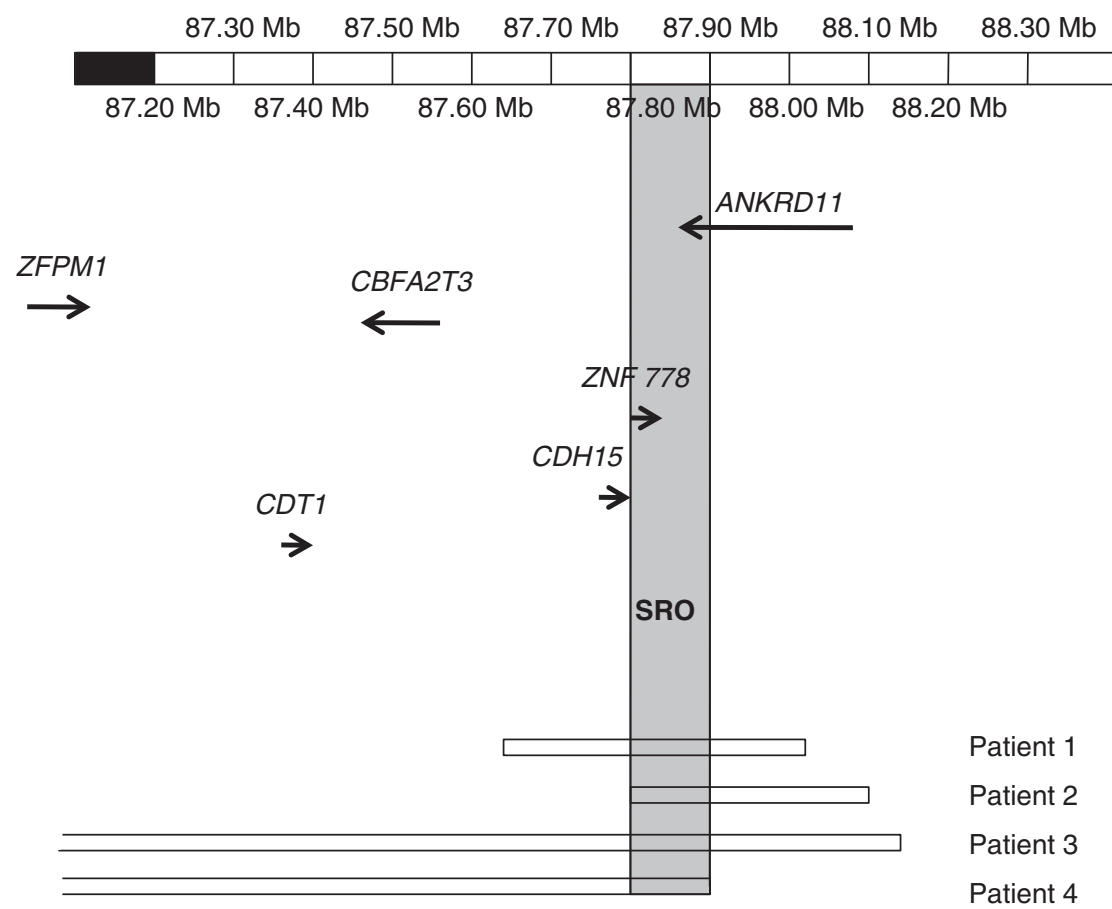

Figure 2 Schematic overview of deleted regions on chromosome $16 q 24.3$ in the presented patients. All deletions were mapped according to the USCS genome browser build May 2004. The relative positions of the genes of interest are indicated. The region of $90 \mathrm{~kb}$ overlap is demarcated with a gray zone. The deletions in patient 3 and 4 extend beyond the figure (Mb positions of proximal delineations: 86.06 and 86.79 , respectively). Arrows indicate the direction in which the genes are transcribed. SRO: shortest region of overlap.

speech delay in patient 2. Patient 2 showed a remarkable discrepancy between his verbal and nonverbal capacities in disadvantage of speech/ auditory development. A reverse discrepancy in IQ levels, however less extreme, was reported in patient 4 who had a borderline-to-normal verbal IQ of 81 and a mildly impaired nonverbal IQ of 67.

Remarkably, both patients 1 and 3 have structural brain abnormalities, including hypoplasia of corpus callosum, colpocephaly/dilated ventricles, as well as periventricular heterotopias that might be causative for the epilepsy these two patients experienced. They share a deletion interval comprising ANKRD11, ZNF778 and CDH15, suggesting that haploinsufficiency for one or more of these genes is involved in neuronal migration and causes the gray matter heterotopias in patient 1 and 3. The localization of the heterotopias in patient 1 was likely bilateral, but no further details were available by cerebral imaging for this patient. The heterotopias in patient 3, however, were unilaterally localized, which raises the possibility that this migrational defect was caused by an (in utero) acquired event rather than by a genetic defect. Both patients 2 and 3 had temporary thrombocytopenia, although the neonatal thrombocytopenia in patient 2 was attributed to his mother's ITP. Nonspecific ocular problems were noted in three of the four patients. Features that were only observed in patient 3 (with the largest deleted region) were a congenital heart defect, unilateral cryptorchidism and sensorineural hearing impairment. Patient 2 also carried a $500 \mathrm{~kb}$ de novo gain at 3 p14.2 at the FRA3B (MIM 601153) locus, which may also contribute to his phenotype; however, patient 2 had no other specific features.

By molecular characterization of these four overlapping deletions in 16q24.3, two likely candidate genes for the observed autism phenotype with cognitive impairment were present in the SRO. A third gene, Cadherin 15 (CDH15), is located just proximal to the SRO and is involved in the deletion observed in patients 1, 3 and 4 (Figure 2). $\mathrm{CDH} 15$ does not seem to be involved in the deletion in patient 2 . The possibility that a position effect or disruption of gene regulatory elements could also create a functional null allele for $\mathrm{CDH} 15$ in this patient is not excluded. The potential involvement of $\mathrm{CDH} 15$ is of particular interest, a nonsynonymous variants of this gene have been identified in a cohort of mentally disabled patients. ${ }^{34}$ However, the group of nonaffected controls in this study was relatively small and the segregation of variants in unaffected family members has not been tested consistently nor was it in agreement with the presumed pathogenicity of the detected variant. Of interest, a recently published genome-wide association study among cases with ASD identified common genetic variants on 5 p14.1 that are associated with susceptibility to ASD. The nearby genes, CDH9 (MIM 609974) and CDH10 (MIM 604555), are also members of the Cadherin gene family involved in neuronal cell adhesion. This implicates a role of $\mathrm{CDH} 9$ and $\mathrm{CDH} 10$ and possibly of other members of the Cadherin family in the pathogenesis of ASD by affecting neuronal cell adhesion. ${ }^{35}$

Haploinsufficiency for either or both ANKRD11 and ZNF778 is an attractive explanation for the syndromic autism phenotype, with variable cognitive impairment observed in the four patients. ANKRD11 encodes a member of the family of ankyrin repeat containing cofactors that interacts with p160 nuclear receptor coactivators and histone deacetylases corepressors and inhibits ligand-dependent transcriptional activation of target genes by nuclear receptors. ${ }^{36}$ Therefore, haploinsufficiency of ANKRD11 may disrupt transcription regulation. Disruption of epigenetic processes has been shown to have a role in causing mental retardation. Examples are disruption of a methyl-CpG-binding protein caused by mutations in MECP2 (MIM 30005) in Rett syndrome, mutations in EMHT1 in the 9q subtelomere 
deletion syndrome and mutations in either CREBBP or EP300 in the Rubinstein-Taybi syndrome. In these cases, mental retardation might be caused by epigenetic effects on the expression of genes that are not themselves mutated. ${ }^{37}$

Interestingly, Barberic et $a l^{38}$ described a mouse mutant, named Yoda, with a missense mutation in the Ankrd11 gene. Homozygous knockout Yoda mice are not viable and die during embryogenesis. This suggests that the gene has a crucial function in embryonic development. A major feature of heterozygous mutant mice is reduced bone mineral density. They also show cranio-facial abnormalities, such as shortened snouts, wider skulls, deformed nasal bones and failure of the interfrontal suture to close. Body size is reduced and with aging many Yoda mice developed kyphosis. ${ }^{38}$ Mild kyphoscoliosis was seen only in the oldest patient (patient 1); however, an examination of bone density in this patient showed normal results. On the basis of in silico data, ANKRD11 is widely expressed, including in several brain regions (amygdala, parietal lobe, occipital lobe and cerebellum) and the heart (UCSC Genome Browser, Microarray Expression Data, Unigene).

The other gene localized in the SRO, ZNF778, encodes a member of the extensive family of KRAB-domain zinc finger proteins. On the basis of available in silico data, this gene is also expressed in several tissues, including the brain and heart (Unigene). KRAB-domain zinc finger proteins are found in transcription regulatory complexes, which are directed to the regulatory elements of target genes through the $\mathrm{C} 2 \mathrm{H} 2$ zinc finger domains that recognize specific DNA binding sites. Moreover, several human zinc finger genes have been associated with MR: ZNF41 (MIM 314995), ZNF81 (MIM 314998) and ZNF674
(MIM 300573) with X-linked $\mathrm{MR}^{39-41}$ and ZNF385B (MIM 612344) with the $2 \mathrm{q} 31.2$ deletion syndrome. ${ }^{41}$ Therefore, haploinsufficiency of ZNF778 might also, or exclusively, be involved in the phenotype observed in the reported patients.

Table 2 provides an overview of these and other interesting genes that are involved in nonoverlapping deleted regions. Proposed functions, phenotype in knockout mice (if available), previously reported cases and the possible clinical correlation of haploinsufficiency of these genes with the observed phenotype in our patients are indicated. Interestingly, the deletion interval of patients 2 and 3 with transient thrombocytopenia comprises the gene ZFPM1 (FOG1) (MIM 601950), which is involved in the regulation of the expression of genes during erythroid and megakaryocytic differentiation. On the basis of available data, we were unable to recognize a candidate gene for the congenital heart defect observed in patient 3 .

In conclusion, we hypothesize that ANKRD11 and ZNF778 are strong candidate genes for the observed core phenotype of this novel 16q24.3 microdeletion syndrome comprising autism spectrum disorder, variable cognitive impairment, facial dysmorphism and brain abnormalities, although a role of one or more of the other genes in the deleted regions of individual patients is not excluded. Moreover, the involvement of ANKRD11 and ZNF778 warrants further studies searching for ANKRD11 and/or ZNF778 mutations in patients with a similar phenotype, but without 16q24 abnormalities by molecular karyotyping. Follow-up of known patients might give answers to the possible occurrence of other phenotypic features. Moreover, the identification of new cases with overlapping deletions is needed to

Table 2 Genes of interest in the entire reported 16q24.3 deletion interval

\begin{tabular}{|c|c|c|c|c|}
\hline positions) & Function & Mouse null & Mouse heterozygous & Previously reported case \\
\hline $\begin{array}{l}\text { ANKRD11 } \\
(87.86-88.08)\end{array}$ & $\begin{array}{l}\text { Nuclear receptor-mediated } \\
\text { transcriptional regulation } \\
\text { (inhibitory) }{ }^{36,38}\end{array}$ & Lethal $^{38}$ & $\begin{array}{l}\text { Reduced bone } \\
\text { mineral density } \\
\text { craniofacial } \\
\text { abnormalies } \\
\text { reduced body size } 38\end{array}$ & $\begin{array}{l}\text { Marshall et al identified } \\
16 q 24.3 \text { deletion encon } \\
\text { passing } A N K R D 11 \text { in an } \\
\text { ASD case. }{ }^{30}\end{array}$ \\
\hline
\end{tabular}

ZNF778

(87.81-87.82)

CDH15

(87.77-87.79)

ZFPM1 (FOG1)

(87.05-87.13)

CBFA2T3 (MIM

603870)

(87.47-87.57)

CDT1

(87.39-87.40)
Encodes a member of the KRAB domain containing zinc finger protein family, suggesting involvement in transcription regulation. 39,42

Cell-cell adhesion in skeletal muscle and human brain. 34,43

\section{Co-regulator of the} transcriptional activator GATA1, which regulates the expression of certain genes during erythroid and megakaryocytic differentiation. 44

Regulates the proliferation and the differentiation of erythroid progenitors Transcriptional regulator (initiation) $^{45}$
No data available No data available

Several other human zinc finger genes have been associated with mental retardation. 39,42

No data available

No data available

GATA-1 knockout mice are not viable. Mice with selective loss of GATA-1 expression in megakaryocytes have thrombopenia.

No data available

No data available
No data available

No data available

No data available

Bhalla et al identified 4 nonsynonymous variants of CDH15 in a cohort of patients with mental retardation. ${ }^{34}$

Freson et al demonstrated a disturbed interaction between mutants of GATA1 and FOG 1 in patients with macrothrombocytopenia and dyserythropoiesis. ${ }^{44}$

No data available
Possible clinical correlate

Kyphoscoliosis in patient 1 , however he has a normal bone density. Syndromic autism phenotype with variable cognitive impairment in patients 1, 2, 3 and 4 Syndromic autism phenotype with variable cognitive impairment in patients $1,2,3$ and 4

Cognitive impairment in patient 1,3 and 4

Neonatal thrombocytopenia in patient 2 and 3 unexplained macrocytosis in patient 3

Unexplained macrocytosis and neonatal thrombocytopenia in patient 3 ? 
extend phenotypic knowledge and to confirm or even further narrow down the critical region of involved genes.

\section{CONFLICT OF INTEREST}

The authors declare no conflict of interest.

\section{ACKNOWLEDGEMENTS}

We thank the parents of patients who participated in this study. This work was supported by grants from the Consortium VG Oost-Nederland (to TK and MHW) and the Genome Canada/Ontario Genomics Institute (to SWS and BF). CRM is supported by the SickKids Foundation and the National Alliance for Research on Schizophrenia and Depression (NARSAD). SWS holds the GlaxoSmithKline-CIHR Pathfinder Chair in Genetics and Genomics at the University of Toronto and Hospital for Sick Children. Patient consent was obtained from the patient's parents for publishing patient pictures in Figure 1. We also thank Dr Zhinshuo Ou and the Kleberg Cytogenetics Laboratory at Baylor College of Medicine.

\section{RESOURCES}

Database of Genomic Variants http://projects.tcag.ca/variation/; Decipher ${ }^{v 41}$ Database of Chromosomal Imbalance and Phenotype in Humans using Ensembl Resources https://decipher.sanger.ac.uk/ application/; European Cytogeneticists Association Register of Unbalanced Chromosome Aberrations (ECARUCA) http://ecaruca. net; Ensembl genome browser http://www.ensembl.org; Unigene http://www.ncbi.nlm.nih.gov/UniGene; USC Genome Browser (Assembly May 2004): http://genome.ucsc.edu; UniProt KB, Assembly July 2008 http://www.uniprot.org.

1 Feuk L, Carson AR, Scherer SW: Structural variation in the human genome. Nat Rev Genet 2006; 7: 85-97.

2 Veltman JA: Genomic microarrays in clinical diagnosis. Curr Opin Pediatr 2006; 18 598-603.

3 Zahir F, Friedman JM: The Impact of array genomic hybridization on mental retardation research: a review of current technologies and their clinical utility. Clin Genet 2007; 72: 271-287.

4 Friedman JM, Baross A, Delaney AD et al: Oligonucleotide microarray analysis of genomic imbalance in children with mental retardation. Am J Hum Genet 2006; 79 500-513.

5 Koolen DA, Sharp AJ, Hurst JA et al: Clinical and molecular delineation of the 17q21.31 microdeletion syndrome. J Med Genet 2008; 45: 710-720.

6 Slavotinek AM: Novel microdeletion syndromes detected by chromosome microarrays. Hum Genet 2008; 124: 1-17.

7 De Vries BBA, Pfundt R, Leisink $M$ et al: Diagnostic genome profiling in mental retardation. Am J Hum Genet 2005; 77: 606-616.

8 Fan Y, Jayakar P, Zhu H et al: Detection of pathogenic gene copy number variations in patients with mental retardation by genomewide oligonucleotide array comparative genomic hybridization. Hum Mut 2007; 28: 1124-1132.

9 Shaffer LG, Theisen A, Bejjani BA et al: The discovery of microdeletion syndromes in the post-genomic era: review of the methodology and characterization of a new 1q41q42 microdeletion syndrome. Genet Med 2007; 9: 607-616.

10 Somerville MJ, Mervis CB, Young EJ et al: Severe expressive-language delay related to duplication of the Williams-Beuren locus. N Engl J Med 2005; 353: 1694-1701.

11 Berg JS, Brunetti-Pierri N, Peters SU et al: Speech delay and autism spectrum behaviors are frequently associated with duplication of the $7 q 11.23$ Williams-Beuren syndrome region. Genet Med 2007; 9: 427-441.

12 Potocki L, Bi W, Treadwell-Deering D et al: Characterization of Potocki-Lupski syndrome (dup(17)(p11.2p11.2)) and delineation of a dosage-sensitive critical interval that can convey an autism phenotype. Am J Hum Genet 2007; 80: 633-649.

13 Zweier C, Peippo MM, Hoyer J et al: Haploinsufficiency of TCF4 causes syndromal mental retardation with intermittent hyperventilation (Pitt-Hopkins Syndrome). A J Hum Genet 2007; 80: 994-1001.

14 Kleefstra T, Brunner HG, Amiel J et al: Loss-of- function mutations in Euchromatin Histone Methyl Transferase 1 (EHMT1) cause the 9q34 subtelomeric deletion syndrome. Am J Hum Genet 2006; 79: 370-777.

15 Petrij F, Giles RH, Dauwerse HG et al: Rubinstein-Taybi syndrome caused by mutations in the transcriptional co-activator CBP. Nature 1995; 376: 348-351.
16 Roelfsema JH, White SJ, Ariyürek Y et al: Genetic heterogeneity in Rubinstein-Taybi syndrome: mutations in both the CBP and EP300 genes cause disease. Am J Hum Genet 2005; 76: 572-580.

17 Vissers LE, van Ravenswaaij CM, Admiraal R et al: Mutations in a new member of chromodomain gene family cause CHARGE syndrome. Nat Genet 2004; 36 : 955-957.

18 Ariani F, Hayek G, Rondinella D et al: FOXG1 is responsible for the congenital variant of Rett syndrome. Am J Hum Genet 2008; 83: 89-93.

19 Conte RA, Kleyman SM, Kharode C, Verma RS: Delineation of a ring chromosome 16 by the FISH-technique: a case report with review. Clin genet 1997; 51: 196-199.

20 Ionasescu V, Patil S, Hart M, Rhead W, Smith W: Multiple congenital anomalies syndrome with myopathy in chromosome 16 abnormality. Am J Med Genet 1987; 26 : 189-194.

21 Ohdo S, Madokoro H, Sonoda T, Ohba K: Sibs lacking characteristic features of duplication of distal 17q. J Med Genet 1989; 26: 465-468.

22 Rack KA, Harris PC, MacCarthy AB et al: Characterization of three de novo derivative chromosomes 16 by 'reverse chromosome painting' and molecular analysis. Am J Hum Genet 1993; 52: 987-997.

23 Ravnan JB, Tepperberg JH, Papenhausen P et al: Subtelomere FISH analysis of 11 688 cases: an evaluation of the frequency and pattern of subtelomere rearrangements in individuals with developmental disabilities. J Med Genet 2006; 43: 478-489.

24 Rossi E, Piccini F, Zollino $\mathrm{M}$ et al: Cryptic telomeric rearrangements in subjects with mental retardation associated with dysmorphism and congenital malformations. J Med Genet 2001; 38: 417-420.

25 Schinzel A: Catalogue of unbalanced chromosome aberrations in man; in de Gruyter (eds): Berlin, 2nd edn, 2001, pp 73-677.

26 Soltan HC, Jung JH, Pyatt Z, Singh R: Partial trisomy 9q resulting from a familial translocation $\mathrm{t}(9 ; 16)(\mathrm{q} 32 ; \mathrm{q} 24)$. Clin Genet 1984; 25: 449-454.

27 Werner W, Kraft S, Callen DF, Bartsch O, Hinkel GK: A small deletion of 16q23.1 $\rightarrow$ $16 q 24.2$ in a boy with iris coloboma and minor anomalies. Am J Med Genet 1997; 70: $371-376$.

28 Yamamoto T, Dowa Y, Ueda $\mathrm{H}$ et al: Tetralogy of Fallot Associated With Pulmonary Atresia and Major Aortopulmonary Collateral Arteries in a Patient With Interstitial Deletion of 16q21-q22.1. Am J Med Genet Part A 2008; 146A: 1575-1580.

29 De Vries BBA, Winter R, Schinzel A, van Ravenswaaij-Arts C: Telomeres: a diagnosis at the end of the chromosomes. J Med Genet 2003; 40: 385-398.

30 Marshall CR, Noor A, Vincent JB et al: Structural Variation of Chromosomes in Autism Spectrum Disorder. Am J Hum Genet 2008; 82: 477-488.

31 Pinto D, Marshall C, Feuk L, Scherer SW: Copy-number variation in control population cohorts. Hum Mol Genet 2007; 16: R168-R173.

32 lafrate AJ, Feuk L, Rivera MN et al: Detection of large-scale variation in the human genome. Nat Genet 2004; 36: 949-951.

33 Redon R, Ishikawa S, Fitch KR et al: Global variation in copy number in the human genome. Nature 2006; 444: 444-454.

34 Bhalla K, Luo Y, Buchan T et al: Alterations in CDH15 and KIRREL3 in patients with mild to severe intellectual disability. Am J Hum Genet 2008; 83: 703-713.

35 Wang $\mathrm{K}$, Zhang $\mathrm{H}$, Ma D et al: Common genetic variants on $5 \mathrm{p} 14.1$ associate with autism spectrum disorders. Nature 2009; 459: 528-533.

36 Zhang A, Li C, Don Chen J: Characterization of transcriptional regulatory domains of ankyrin repeat cofactor-1. Biochem and Biophys Research Comm 2007; 358: 1034-1040.

37 Grant ME: The epigenetic origins of mental retardation. DNA methylation and mental retardation. Loss of dynamic histone regulation underlies mental retardation. Clin Genet 2008; 73: 528-534.

38 Barberic I, Perry MJ, Dear TN et al: An ENU-induced mutation in the Ankrd11 gene results in an osteopenia-like phenotype in the mouse mutant Yoda. Physiol. Genomics 2008; 32: 311-321.

39 Shoichet SA, Hoffmann K, Menzel $\mathrm{C}$ et al: Mutations in the ZNF41 gene are associated with cognitive deficits: identification of a new candidate for X-linked mental retardation. Am J Hum Genet 2003; 73: 1341-1354.

40 Kleefstra T, Yntema HG, Oudakker AR et al: Zinc finger 81 (ZNF81) mutations associated with X-linked mental retardation. J Med Genet 2004; 41: 394-399.

41 Lugtenberg D, Yntema HG, Banning MJG et al: ZNF674: a new Kruppel-associated box-containing zinc-finger gene involved in nonsyndromic X-linked mental retardation. Am J Hum Genet 2006; 78: 265-278.

42 Monfort S, Rosello M, Orellana C et al: Detection of known and novel genomic rearrangements by array based comparative genomic hybridisation: deletion of ZNF533 and duplication of CHARGE syndrome genes. J Med Genet 2008; 45: 432-437.

43 Shimoyama Y, Tsujimoto G, Kitajima M, Natori M: Identification of three human type-II classic cadherins and frequent heterophilic interactions between different subclasses of type-II classic cadherins. Biochem J 2000; 349: 159-167.

44 Freson K, Thys C, Wittewrongel C, Vermylen J, Hoylaerts MF, Van Geet C: Molecular cloning and characterization of the GATA1 cofactor human FOG1 and assessment of its binding to GATA1 proteins carrying D218 substitutions. Hum Genet 2003; 112 $42-49$

45 Rialland M, Sola F, Santocanale C: Essential role of human CDT1 in DNA replication and chromatin licensing. J Cell Sci 2002; 115: 1435-1440. 